\title{
Bell-inequality and two slit experiments: Comparing misapplication of classical probability by Feynman and Bell
}

\author{
Andrei Khrennikov \\ Linnaeus University, International Center for Mathematical Modeling \\ in Physics and Cognitive Sciences Växjö, SE-351 95, Sweden
}

June 24, 2021

\begin{abstract}
We start with the discussion on misapplication of classical probability theory by Feynman in his analysis of the two slit experiment (by following the critical argumentation of Koopman, Ballentine, and the author of this paper). The seed of Feynman's conclusion on the impossibility to apply the classical probabilistic description for the two slit experiment is treatment of conditional probabilities corresponding to different experimental contexts as unconditional ones. Then we move to the Bell type inequalities. Bell applied classical probability theory in the same manner as Feynman and, as can be expected, he also obtained the impossibility statement. In contrast to Feynman, he formulated his no-go statement not in the probabilistic terms, but by appealing to nonlocality. This note can be considered as a part of the author's attempts for getting rid off nonlocality from quantum physics.
\end{abstract}

\section{Introduction}

Nowadays quantum nonlocality which was highlighted by Bell [1] (see also, e.g., [2]-[6]) is the basic component of modern quantum physics (in contrast to "good old quantum physics" of Planck, Einstein, Bohr, Heisenberg, Pauli, Fock, Landau, Blohintzev etc.). However, this nonlocality is apparent and appeal to it in the foundational discussions is really misleading. 
Since 1990-s, I tried to get rid off nonlocality from quantum physics. Initially the purely probabilistic reasoning was used - to show that Bell misapplied of the classical probability theory $(\mathrm{CP})$ in his derivation of the famous inequality (e.g., [7] - [9]; see also Kupczynski [10]). Recently I directly appealed to the formalism of quantum theory by coupling the Bell type inequalities with incompatibility of quantum observables [11]-[15] (see also Boughn [16]).

This paper is coupled to my previous works on CP-misapplication in Bell's argumentation, especially to recent papers [17, 18], where the role of conditional probability (defined classically with the Bayes formula) was highlighted. The starting point is the critique of Feynman's probabilistic analysis of the two slit experiment [19, 20]. Feynman tried to apply CP to model the experimental output (as well as the prediction by quantum theory) of the two slit experiment. However, he misapplied CP by treating conditional probabilities corresponding to variety of experimental contexts as unconditional so to say absolute probabilities. This led him to the conclusion on incompatibility of $\mathrm{CP}$ with quantum theory and experiment. This problem in Feynman's probabilistic reasoning is well known for experts (see e.g. Koopman [21], Ballentine [22], and Khrennikov [7, 9, 23]) and this is the good time to recall about it.

Later Feynman-like reasoning was used by Bell. We analyze parallelism of Feynman and Bell reasoning generating the apparent contradiction between $\mathrm{CP}$ and quantum physics. We point that, in contrast to Feynman, Bell overshadowed this conclusion by nonlocality issue.

\section{Classical probability theory}

CP was mathematically formalized by Kolmogorov [24] (1933). This is the calculus of probability measures, non-negative weight $P(A)$ is assigned to any event $A$; here $0 \leq P(A) \leq 1$.

The main property of $\mathrm{CP}$ is its additivity: if two events $A_{1}, A_{2}$ are disjoint, then the probability of disjunction of these events equals to the sum of probabilities:

$$
P\left(A_{1} \vee A_{2}\right)=P\left(A_{1}\right)+P\left(A_{2}\right) .
$$

To make theory mathematically interesting, condition of additivity is extended to countable-additivity.

By using Bayes' formula

$$
P(A \mid C)=P(A \mid C) / P(C),
$$

there is introduced the conditional probability $P(A \mid C)$ : the probability that event $A$ would happen under the condition that event $C$ was 
happened. We are interested in the situation that event $C$ is selection of a complex of experimental conditions, context $C$. The conditional probability $A \rightarrow P_{C}(A) \equiv P(A \mid C)$ is also a probability measure; hence it is also additive:

$$
P\left(A_{1} \vee A_{2} \mid C\right)=P\left(A_{1} \mid C\right)+P\left(A_{2} \mid C\right),
$$

for disjoint events.

Consider now a random variable which represents some observable $O$, then, for each value $\alpha$, and any two disjoint events $C_{1}$ and $C_{2}$, we have, for event $C_{12}=C_{1} \vee C_{2}$,

$$
P\left((O=\alpha) \wedge C_{12}\right)=P\left((O=\alpha) \wedge C_{1}\right)+P\left((O=\alpha) \wedge C_{2}\right) .
$$

However, generally

$$
P\left(O=\alpha \mid C_{12}\right)=P\left(O=\alpha \mid C_{1} \vee C_{2}\right) \neq P\left(O=\alpha \mid C_{1}\right)+P\left(O=\alpha \mid C_{2}\right) .
$$

So, the calculus of conditional probabilities differs from calculus of " "absolute probabilities".

\section{Probabilistic analysis of the two slit experiment: Feynman versus Koopman, Ballentine, Khrennikov}

Here we follow Ballentine [22] (see also Koopman [21] and Khrennikov $[7,9,23])$. The two slit experiment can be described as follows. There is a source, a screen with two slits (labeled $s 1$ and $s 2$ ) in it, and a detector. The detector can be moved to measure the particle count rate at various positions. In this way, an experiment can measure the probability of a particle passing through the slit system and arriving at the point, $x$. Consider the experimental context $C_{1}$ such that only slit $s 1$ is open; the probability of detection at point $x$ is denoted by $P_{1}$. (Point $x$ is fixed, so we omit it form notation.) Consider also another experimental context $C_{2}$ such that only slit $s 2$ is open; the probability of detection at point $x$ is denoted by $P_{2}$. Finally, consider the experimental context $C_{12}$ such that both slits are open, the probability of detection is $P_{12}$.

We note that passage of a particle through slit $s 1$ and passage through slit $s 2$ are certainly exclusive events. Hence events of passing through $C_{1}$ and $C_{2}$ are disjoint and event $C_{12}$ of passing either through $C_{1}$ or $C_{2}$, when both slits are open, is disjunction of these events. 
Hence, one might expect (as Feynman did [19, 20]) from additivity of probability (1) and concretely from (3), that $P_{12}$ should be equal to $P_{1}+P_{2}$. But, as is well known, the experiment statistical data shows that this is not true. Hence, it might be concluded that of probability theory does not hold in quantum mechanics.

Ballentine pointed out [22]: "In fact, the above argument draws its radical conclusion from an incorrect application of probability theory." He had in mind that Feynman misleadingly appealed to the formula (1), instead of (4). To make this issue clearer, we introduce the observable $O \equiv O x$ representing the clicks of a detector located at the point $x$ and yielding the values 0 (no click) and 1 (click). In this notation, $P_{i} \equiv P_{i}(O=+1)$ and $1-P_{i}=P_{i}(O=0)$, as well as $P_{12} \equiv P_{12}(O=+1), 1-P_{12}=P_{12}(O=0)$. Feynman's probability fallacy can be written as

$$
P_{12}(O=\alpha) \neq P_{1}(O=\alpha)+P_{2}(O=\alpha), \alpha=0,1 .
$$

But, in the CP-framework there is no reason to expect that

$$
P\left(O=\alpha \mid C_{12}\right)=P\left(O=\alpha \mid C_{1}\right)+P_{2}\left(O=\alpha \mid C_{2}\right), \alpha=0,1 .
$$

\section{Feynman-like reasoning of Bell}

The Bell experiment can be described as follows. There is a source of pairs of particles and two polarization beam splitters (PBSs) with orientations $\theta=\left(\theta_{A}, \theta_{B}\right)$; each PBS is coupled to a pair of detectors, $D_{A}( \pm), D_{B}( \pm)$. Pairing of detector's outputs for Alice and Bob can be represented as observable $O$ taking vector-values $(\alpha, \beta)$, where $\alpha, \beta= \pm 1$. Now to create the CHSH-combination of correlations, experimenter has to consider four experimental contexts, corresponding to selection of two Alice's angles $\theta_{A 1}, \theta_{A 2}$ and two Bob's angles $\theta_{B 1}, \theta_{B 2}$. We denote the corresponding contexts as $C_{i j}, i, j=1,2$. Then correlations are combined of probabilities $P\left(O=(\alpha, \beta) \mid C_{i j}\right)$. We have

$$
\begin{aligned}
\mathcal{C H S H} & =\sum_{i, j=1,2} \sigma_{i j}\left(\left[P\left(O=(+1,+1) \mid C_{i j}\right)+P\left(O=(-1,-1) \mid C_{i j}\right)\right]\right. \\
& \left.-\left[P\left(O=(+1,-1) \mid C_{i j}\right)+P\left(O=(-1,+1) \mid C_{i j}\right)\right]\right),
\end{aligned}
$$

where just one of $\sigma_{i j}$ equals -1 , others equal +1 . This consistent context-referring of probabilities prevents derivation of the CHSH inequality,

$$
|\mathcal{C H S H}| \leq 2
$$


Therefore it is not surprising that it is violated for the experimental data.

In fact, this is the same probability fallacy - mixing of conditional and unconditional probabilities. To derive (8), one has to consider unconditional probabilities and the corresponding CHSH-combination of correlations.

\section{Concluding remarks}

The violation of the Bell inequality is the hottest topic of the modern foundational debates. They are characterized by the diversity of the mutually contradicting interpretations. In this paper I presented the following interpretation:

Bell as well as Feynman "simply" confused conditional and unconditional probabilities. In this way, they found that CP (and hence classical physics which is based on CP) contradicts to quantum theory. In contrast to Feynman, Bell did not formalize his conclusion in the purely probabilistic terms. This led him to coupling the purely probabilistic interplay between conditional and unconditional probabilities to the issue of nonlocality.

In short, this interpretation can be formulated as follows:

In the framework of multi-contextual experiment (as the Bell type experiments: 4 different contexts for the CHSH test), generally there is no Bell inequality, so there is nothing to violate.

Hence, Bell's appealing to quantum nonlocality is misleading.

acknowledgments: The paper was published with the financial support of the Ministry of Education and Science of the Russian Federation as part of the program of the Mathematical Center for Fundamental and Applied Mathematics under the agreement N 075-152019-1621.

\section{References}

[1] Bell, J.S. On the Einstein-Podolsky-Rosen paradox. Physics 1964, 1, 195-200

[2] Bell, J.S. Speakable and Unspeakable in Quantum Mechanics, 2nd ed.; Cambridge University Press: Cambridge, UK, 2004.

[3] Bell, J.S. On the problem of hidden variables in quantum theory. Rev. Mod. Phys. 1966, 38, 450. 
[4] Clauser, J.F.; Horne, M.A.; Shimony, A.; Holt, R.A. Proposed experiment to test local hidden-variable theories. Phys. Rev. Lett. 1969, 23, 880.

[5] A. Aspect, Experimental tests of Bell's inequalities in atomic physics, in Atomic Physics 8, Proceedings of the Eighth International Conference on Atomic Physics, edited by I. Lindgren, A. Rosen and S. Svanberg (1982).

[6] A. Aspect, Bell's Theorem: The naive view of an experimentalist. quant-ph/0402001.

[7] Khrennikov, A. Yu. (1999). Interpretations of Probability (VSP Int. Sc. Publishers, Utrecht/Tokyo); 2nd edn. (De Gruyter, Berlin, 2009).

[8] Khrennikov, A. Non-Kolmogorov probability models and modified Bell's inequality, J. Math. Phys. 2000, 41, 1768-1777.

[9] Khrennikov, A. (2009). Contextual Approach to Quantum Formalism, (Springer, Berlin-Heidelberg-New York).

[10] Kupczynski, M. Bertrand's paradox and Bell's inequalities. Phys. Lett. A 1987, 121, 205-207.

[11] A. Khrennikov, Get rid of nonlocality from quantum physics. Entropy, 21(8), 806 (2019).

[12] A. Khrennikov, Bell argument: Locality or realism? Time to make the choice. AIP Conf. Proc., 1424, pp. 160-175 (2012); https://arxiv.org/pdf/1108.0001v2.pdf

[13] A. Khrennikov, Bohr against Bell: complementarity versus nonlocality. Open Phys. 15, 734-73 (2017).

[14] A. Khrennikov, Quantum versus classical entanglement: eliminating the issue of quantum nonlocality. Found Phys 50, 1762-1780 (2020).

[15] A. Khrennikov, Two faced Janus of quantum nonlocality. Entropy 2020, 22, 303; arXiv:2001.02977 [quant-ph].

[16] Boughn, S. Making sense of Bell's theorem and quantum nonlocality. Found. Phys. 2017, 47, 640-657.

[17] Khrennikov, A. CHSH inequality: quantum probabilities as classical conditional probabilities. Found. Phys. 2015, 45, 711-725.

[18] Khrennikov, A.; Alodjants, A. Classical (local and contextual) probability model for Bohm-Bell type experiments: no-signaling as independence of random variables, Entropy 2018, 21, 157. 
[19] Feynman P. The concept of probability in quantum mechanics. In Proceedings of the Second Berkeley Symposium on Mathematical Statistics and Probability, 1951, pp. 553-541. Univ. of California Press. Berkeley, California

[20] Feynman, R. and Hibbs, A.: Quantum Mechanics and Path Integrals. McGraw-Hill, New York (1965)

[21] Koopman, . 0. 1955. Quantum theory and the foundations of probability. In Applied Probability. L. A. MacColl, Ed.: 97-102. McGraw-Hill. New York.

[22] L. Ballentine, Probability in Quantum Mechanics. Annals of New York Academy of Science, Techniques and Ideas in Quantum Measurement Theory, 480, N 1, 382-392 (1986).

[23] Khrennikov, A. (2005). The principle of supplementarity: A contextual probabilistic viewpoint to complementarity, the interference of probabilities, and the incompatibility of variables in quantum mechanics, Found. Phys., 35, N 10, pp. 1655-1693.

[24] Kolmolgoroff, A. N. (1933). Grundbegriffe der Wahrscheinlichkeitsrechnung, (Springer-Verlag, Berlin). 